Trinity University

Digital Commons @ Trinity

Psychology Faculty Research

Psychology Department

$6-2011$

\title{
A Comparison of Mindfulness, Nonjudgmental, and Cognitive Dissonance-Based Approaches to Mirror Exposure
}

Cynthia A. Luethcke

Trinity University, luethcke@gmail.com

Leda McDaniel

Trinity University

Carolyn Becker

Trinity University, cbecker@trinity.edu

Follow this and additional works at: https://digitalcommons.trinity.edu/psych_faculty

Part of the Psychology Commons

Publication Details

Body Image

\section{Repository Citation}

Luethcke, C.A., McDaniel, L., \& Becker, C.B. (2011). A comparison of mindfulness, nonjudgmental, and cognitive dissonance-based approaches to mirror exposure. Body Image, 8(3), 251-258. doi: 10.1016/ j.bodyim.2011.03.006

This Post-Print is brought to you for free and open access by the Psychology Department at Digital Commons @ Trinity. It has been accepted for inclusion in Psychology Faculty Research by an authorized administrator of Digital Commons@ Trinity. For more information, please contact jcostanz@trinity.edu. 
A Comparison of Mindfulness, Nonjudgmental, and Cognitive Dissonance-Based Approaches to Mirror Exposure

Cynthia A. Luethckea, B.A., Leda McDaniela , B.A., \& Carolyn Black Becker ${ }^{a}$, Ph.D.

a Trinity University

One Trinity Place

San Antonio, TX 78212

in press Body Image: Do not cite or distribute without permission of authors

Correspondence: Cynthia Luethcke

Email: luethcke@gmail.com

1 Present Address: Department of Psychiatry - Mail Code 7747, University of Texas Health Science Center at San Antonio, 7550 IH 10 West, Suite 1325, San Antonio, TX 78229 


\begin{abstract}
This study compares different versions of mirror exposure (ME), a body image intervention with research support. ME protocols were adapted to maximize control and comparability, and scripted for delivery by research assistants. Female undergraduates $(N=168)$ were randomly assigned to receive mindfulness-based $(\mathrm{MB} ; n=58)$, nonjudgmental $(\mathrm{NJ} ; n=$ 55), or cognitive dissonance-based (CD, $n=55)$ ME. Participants completed the Body Image Avoidance Questionnaire (BIAQ), Body Checking Questionnaire (BCQ), Satisfaction with Body Parts Scale (SBPS), Beck Depression Inventory-II (BDI-II), and Eating Disorders Examination Questionnaire (EDE-Q) at pre-treatment, post-treatment, and 1-month follow-up. Mixed models ANOVAs revealed a significant main effect of time on all measures, and no significant time by condition interaction for any measures except the SBPS. Post-hoc analysis revealed that only CD ME significantly improved SBPS outcome. Results suggest that all versions of ME reduce eating disorder risk factors, but only CD ME improves body satisfaction.
\end{abstract}

Keywords: Body Image, Body Satisfaction, Mirror Exposure, Mindfulness, Cognitive Dissonance, Eating Disorders 
A Comparison of Mindfulness, Nonjudgmental, and Cognitive Dissonance-Based Approaches to Mirror Exposure

Body image disturbance is viewed as the core psychopathology for eating disorders such as bulimia nervosa and anorexia nervosa $(D S M-I V-T R, 2000)$. It is the key factor that sets eating disorders apart from other psychological disorders that occasionally involve abnormal eating or weight loss (Rosen, 1990), and it plays a central role in the onset (Killen et al., 1996; Stice, 2002), maintenance (Fairburn et al., 2003; Stice, 2002), and relapse of eating disorders (Fairburn, Peveler, Jones, Hope \& Doll, 1993; Freeman, Beach, Davis \& Solym, 1985). Research has documented residual body image disturbance in post-treatment follow-up of weight-recovered anorexic patients (Widenauer, Lennerts, Talbot, Touyz, \& Beumont, 1993), and in patients with bulimia nervosa after receiving empirically supported therapies (Fairburn et al., 1993). Treating body image disturbance should therefore be a central focus of therapy and research.

Many researchers have investigated mirror exposure (ME) as a possible treatment for body image disturbance (Key et al., 2002; Delinsky \& Wilson, 2006; Hilbert, Tuschen-Caffier \& Vögele, 2002; Jansen et al., 2008). Research suggests that incorporating ME into body image treatment enhances outcomes for anxiety, avoidance behaviors, and body dissatisfaction (Key et al., 2002). During ME, clients observe their bodies in a mirror and focus on different parts of their body non-critically and for equal amounts of time. This exercise is usually guided by a comprehensive list of body parts or areas of the body to address. Providing such a list prevents clients from dismissing certain areas that they may be uncomfortable with and might otherwise avoid during the mirror exposure exercise.

One suggested model for ME proposes that ME works by extinguishing a conditioned relationship between the body and feelings of disgust or anxiety (Jansen et al., 2008). Jansen et 
al. postulated that the body may act as the conditioned stimulus and, after being paired repeatedly with the unconditioned stimulus of negative body-related thoughts, becomes linked to negative feelings such as anxiety and disgust. Thus, subsequent exposure to the body alone could directly cause negative feelings. ME might work by exposing clients to their body in the absence of negative body-related thoughts, thereby extinguishing the relationship between the image of the body and negative emotions (Jansen et al., 2008). Previous studies have not validated this model in full, though research indicates that negative emotional reactivity and body-related cognitions are significantly reduced in both non-clinical and eating disordered participants during ME (Vocks, Legenbauer, Wächter, Wucherer, \& Kosfelder, 2007).

Various types of mirror exposure have been used in previous research, including a cognitive dissonance-based ME (CD ME), a neutral ME (in this paper, referred to as a nonjudgmental ME; NJ ME), and a mindfulness-based ME (MB ME). Stice and Presnell (2007) incorporated a CD ME into their cognitive dissonance-based eating disorders prevention program. In their CD ME, participants described positive aspects of their physical, emotional, intellectual, and social qualities while looking at their image in a mirror. For an individual with body dissatisfaction, the act of complementing his or her self while looking in the mirror should induce cognitive dissonance. Though several studies have documented the effectiveness of cognitive dissonance-based prevention programs in reducing risk factors for eating disorders (Stice, Shaw, Becker, \& Rhode, 2008), the specific contribution of CD ME to this effect remains to be determined.

In addition to $\mathrm{CD} \mathrm{ME}$, researchers have recently studied a mindfulness-based version of ME (Delinsky \& Wilson, 2006). An essential component of mindfulness is maintaining a nonjudgmental frame of mind, which is incompatible with the perspective of someone with body 
dissatisfaction because these individuals primarily hold a negative viewpoint (Stewart, 2004). A mindfulness-based treatment may therefore be particularly well suited for treating body image disturbance. In the MB ME used by Delinsky and Wilson, participants were encouraged to engage in a state of mindfulness while viewing their bodies in a mirror and making nonjudgmental observations about each body part aloud. When compared with a nondirective body image treatment control group, Delinsky and Wilson found that the MB ME intervention led to significantly greater improvements in body checking, body avoidance, weight and shape concerns, depression, and self-esteem (Delinsky \& Wilson, 2006).

Mindfulness may be sufficient for body image improvement, but all of its components may not be necessary. Research suggests that a nonjudgmental stance alone during ME is effective in reducing body dissatisfaction. Hilbert et al. (2002) tested the effectiveness of a neutral, or nonjudgmental (NJ), ME. ${ }^{1}$ In this ME exercise, participants answered a series of questions about their appearance by describing their body "as precisely and neutrally as possible" (Hilbert et al., 2002, p. 138). Research suggests that this type of NJ ME reduces negative cognitions and mood, and increases appearance-related self-esteem in a non-clinical group and in a group with binge-eating disorder (Hilbert et al., 2002). Preliminary data also suggests that this type of ME reduces anxiety and improves body satisfaction in obese adolescents (Jansen et al., 2008).

Though various approaches to ME have been used in isolated studies, the effectiveness of these approaches has not been directly compared. In this study, we hypothesized that using

\footnotetext{
${ }^{1}$ The neutral approach to ME used by Hilbert et al. (2002) is sometimes referred to as a nonjudgmental $(\mathrm{NJ})$ approach in this paper to indicate its similarity to the nonjudgmental aspect of the MB approach.
} 
neutral body descriptions during $\mathrm{ME}$, the approach taken in both $\mathrm{MB}$ and NJ ME, would be more effective than the positive descriptions used in CD ME. Overall, we theorized that it would be more difficult to replace negative body-related cognitions with positive cognitions, rather than neutral cognitions. Voicing positive opinions about one's own appearance directly opposes the social norm of feminine modesty. Previous research suggests that women who speak well of themselves are rated as less socially attractive in situations such as job interviews (Rudman, 1998). Research specifically related to conversation about appearance furthermore suggests that women are expected to engage in fat talk, and are more likely to be accepted by their female friends when they do so (Britton, Martz, Bazzini, Curtin, \& LeaShomb, 2006). Generally speaking, fat talk consists of a self-deprecating conversation between girls or women in which each describes herself as fat while reassuring others that they are thin (Nichter, 2000, p. 45). The positive self-talk used during CD ME would be a direct challenge to the established norms of fat talk and feminine modesty, whereas expressing an objective opinion (i.e., nonjudgmental comments) would not run contrary to social norms. In fact, research suggests that women already have more neutral than positive body-related cognitions (Hilbert \& Tuschen-Caffier, 2005). Hilbert and Tuschen-Caffier (2005) coded the valence of body-related cognitions verbalized in a free-response setting by women (with and without eating disorders) looking at their mirror image, and found that neutral cognitions were more frequent than positive cognitions in both groups. This research supports the idea that women may be more willing to adopt a neutral perspective rather than a positive perspective about their bodies.

The current study investigated the effectiveness of three approaches to mirror exposure, including two neutral approaches, $\mathrm{MB}$ and $\mathrm{NJ}$, and one positive approach, $\mathrm{CD}$. These three versions of ME were selected because previous studies have demonstrated the effectiveness of 
these approaches for improving eating disorder-related variables (Delinsky \& Wilson, 2006; Hilbert et al., 2002; Jansen et al., 2008; Stice \& Presnell, 2007). Consistent with this previous evidence, we hypothesized that all three conditions would reduce eating disorder risk factors. Based on previous research regarding feminine modesty, as well as observed body-related cognitions during ME (Hilbert \& Tuschen-Caffier, 2005), we furthermore hypothesized that the neutral approach used by both the NJ and the MB MEs would result in outcomes superior to CD ME. If NJ ME and MB ME are equally effective, this would suggest that neutrality is the active component of the MB approach.

\section{Materials and Methods}

\section{Participants}

Participants were female undergraduates attending a small liberal arts university in the southern United States. A non-clinical sample was used because previous research comparing the effectiveness of ME in populations with and without eating disorders suggests improvement in both groups (Vocks et al., 2007; Hilbert et al., 2002). Data collected in non-clinical populations also can provide groundwork for eating disorder prevention programs. In order to reduce the chance of a ceiling effect, we attempted to recruit individuals with more body image disturbance by advertising the ME experiment as a program for improving body image.

In the first and second year of the study, first-year female students were recruited via email and poster advertisement, and incentives included two raffles for a \$100 American Express gift card, and participatory credit in introductory psychology courses. In the third year, female students were recruited from introductory psychology classes and only received participatory credit. When appointments were scheduled, experimenters requested that participants refrain 
from eating for two hours prior to ME so as to standardize a variable that may have an effect on feelings of guilt or fatness.

A total of 192 females participated in the study. Out of these participants, 12 participants were excluded from analyses because they did not complete the second session and/or the 1month follow-up, and 1 was excluded because of an error in the experimental procedure. Furthermore, because the aim of this study was to address body image dissatisfaction in a nonclinical population, 6 participants were excluded because they met criteria for anorexia or bulimia nervosa based on their responses to the Eating Disorder Examination-Questionnaire (EDE-Q; Fairburn \& Beglin, 1994). Additionally, participants were excluded if they were visibly pregnant $(n=1)$, or if they met the National Institutes of Health's (1998) definition of obesity (BMI $\geq 30 ; n=4)$. Obese participants were excluded because they may experience higher levels of concern about shape and weight due to a realistic and health-related need to lose weight (rather than internalization of the thin ideal). Out of the final sample of participants $(N=$ 168), the majority were Caucasian (61.9\%) but other ethnicities endorsed were: Hispanic (10.1\%), Asian (7.1\%), African American (1.8\%), Hawaiian or other pacific islander (.6\%), more than one race $(6.5 \%)$, other $(1.8 \%)$, and $10.1 \%$ did not respond. Participants indicating age $(n=$ $153)$ were $17-21$ years of age $(M=18.45, S D=0.78)$.

\section{Measures}

Body Checking Questionnaire (BCQ). Body checking was assessed with the BCQ (Reas, Whisenhunt, Netemeyer, \& Williamson, 2002). This 23-item inventory assesses the frequency (from $1=$ never to $5=$ very often) with which participants engage in body checking behaviors such as "I pinch my stomach to measure fatness." The BCQ has demonstrated good reliability ( $r=.94$; Reas et al., 2002) and the subscales (i.e., overall appearance, specific body 
parts, and idiosyncratic checking) all have good internal consistency $(\alpha=0.84-0.92$; Calugi, Dalle Grave, Ghisi, \& Sanavio, 2006). In the present study, the BCQ also demonstrated excellent internal consistency $(\alpha=.91)$.

Body Image Avoidance Questionnaire (BIAQ). Body image avoidance behaviors were measured with the BIAQ (Rosen, Srebnik, Saltzberg, \& Wendt, 1991). This 19-item questionnaire assesses the extent to which situations or behaviors that could expose body image concerns are avoided (e.g., wearing tight clothing, engaging in physical intimacy, taking part in social activities). Answers are rated on a Likert scale (from $0=$ never, to $4=$ always). This measure has demonstrated good reliability $(r=.87)$ and internal consistency $(\alpha=.89$; Rosen et al., 1991). The BIAQ demonstrated acceptable internal consistency in this study $(\alpha=.78)$.

Satisfaction with Body Parts Scale (SBPS). The SBPS was selected because Delinsky and Wilson (2006) used this measure, and the present study aims, in part, to expand upon their findings. The SBPS rates the degree of satisfaction (from $1=$ extremely dissatisfied, to $6=$ extremely satisfied) with various body parts/areas. Delinsky and Wilson used the shortened, 14item version of the scale. This version of the SBPS has good internal validity and has good concurrent validity with other measures of body image as well as small to medium correlation with eating disorder symptoms (Petrie, Tripp, \& Harvey, 2002). The present study used the longer version of the SBPS (Berscheid, Walster, \& Bohrnstedt, 1973) because it is more comprehensive. One item included in the original scale, voice, was excluded because it was the only item judged to be unrelated to appearance of body parts. The 23-item SBPS used in this study had good internal consistency $(\alpha=.88)$.

Beck Depression Inventory-II (BDI-II). Depression was measured with the BDI-II (Beck, Steer, \& Brown, 1996). Depression was assessed in this study because depression and 
self-esteem problems often co-occur with eating disorders (Wilson \& Fairburn, 2002), and research suggests that ME may play a role in lowering depression in individuals with body image dissatisfaction (Delinsky \& Wilson, 2006). The BDI-II is a well-accepted, self-report assessment of depression as measured by 21 items over the past two weeks. The BDI-II has demonstrated good internal consistency ( $\alpha=.91$; Dozois, Dobson, $\&$ Ahnberg, 1998) and good test-retest reliability (Wiebe \& Penley, 2005). In this study, 20 of the 21 items were used. The question regarding suicide was excluded due to long-standing IRB preference at the institution where this project was run. The 20-item BDI-II used in this study had good internal consistency $(\alpha=.86)$.

Eating Disorder Examination Questionnaire (EDE-Q). The EDE-Q is the self-report, questionnaire version of the Eating Disorder Examination (EDE), the widely used and preferred diagnostic, semi-structured interview for eating disorders. Scores on the EDE-Q and the EDE significantly correlate, though the EDE-Q tends to overestimate more ambiguous features like binge eating and shape concern (Fairburn \& Beglin, 1994). The full EDE-Q has demonstrated good internal consistency ( $\alpha=0.90$; Peterson et al., 2007). This study used two subscales from the EDE-Q $\left(5^{\text {th }}\right.$ ed.), shape concern and weight concern, to generate one overall score for shape and weight concern. Each of these subscales has demonstrated excellent internal consistency in previous research (shape concern, $\alpha=0.83$, and weight concern, $\alpha=0.72$; Peterson et al., 2007). Scores on shape and weight concern subscales were added together and divided by 2 to calculate an overall score. Rationale for combining these scales comes from previous research, which has found that these two subscales are highly correlated $(r=.92)$, and that the internal consistency of the combined scale is excellent ( $\alpha=.94$; Becker et al., 2010). Similarly, in the present study, these two subscales had good correlation $(r=.84)$, and the combined shape and weight concern scale had excellent internal consistency $(\alpha=.92)$. 
Additionally, because the EDE-Q ( $5^{\text {th }}$ ed.) includes 10 eating disorder diagnostic questions, a composite score for eating disorder pathology was generated from these questions. In this study, the eating disorder pathology subscale had good internal consistency $(\alpha=.81)$. The EDE-Q was also used to determine whether participants met DSM-IV-TR criteria for bulimia or anorexia, which excluded them from analysis. The EDE-Q has been used in other studies for this purpose (Becker, Bull, Schaumberg, Cauble, \& Franco, 2008; Becker, Smith, \& Ciao, 2006; Becker, Stice, Shaw, \& Woda, 2009).

\section{Procedures}

Session Timeline and Content. All assessments and interventions were delivered by female research assistants, including psychology undergraduate students and one bachelor'slevel research assistant. The study consisted of three separate sessions. Upon arrival at the first session, a block-randomization procedure was used to assign participants to one of three ME conditions: $\mathrm{MB}(n=58), \mathrm{NJ}(n=55)$, or $\mathrm{CD}(n=55)$. After randomization, participants filled out a baseline questionnaire packet with all measures before engaging in the ME exercise (NJ and $\mathrm{CD}$ conditions) or in a guided mindfulness exercise (MB condition). After this, participants in the MB condition completed the ME exercise. The second session took place approximately one week after the first session. In the second session, participants completed a second ME exercise and then filled out a post-treatment questionnaire packet with all measures. The third session took place approximately one month after the second session. In this session, participants completed a questionnaire packet with all measures and then were debriefed. Questionnaires were randomly ordered and distributed in packets. Since all measures were selfreport, they were completed in a private, quiet room. 
Mindfulness Exercise. Before their first mirror exposure, MB participants were introduced to the concept of mindfulness and completed a beginner's guided breathing meditation exercise from a compact disc series included in Salzberg and Goldstein's Insight Meditation course (2001). This recording was chosen because Salzberg and Goldstein are wellestablished instructors of mindfulness meditation. This exercise was added to the MB condition so that participants could experience and practice mindfulness before being asked to maintain a state of mindfulness during ME. Clients who are learning to practice mindfulness in the context of psychotherapy are often required to spend a significant amount of time practicing mindfulness skills between therapy sessions. Therefore, the MB group was also assigned homework after their first session. Participants in this group were asked to practice mindfulness during an everyday activity (e.g., brushing teeth or walking to class) before their second session. During the second session, participants in the MB condition reported the extent to which they completed their homework on a Likert scale ranging from one to three $(1=$ fully completed, $2=$ somewhat complete, $3=$ did not complete $)$. Participants in the MB group who responded to the question $(n$ $=55)$ indicated moderate homework compliance $(M=1.78 ; S D=0.5){ }^{2}$

Mirror Exposure (ME) Exercise. Participants in the MB condition completed the ME exercise after their mindfulness exercise, while participants in the $\mathrm{NJ}$ and $\mathrm{CD}$ conditions were given instructions for the ME exercise immediately following completion of baseline questionnaires. Mirror exposures in all conditions consisted of participants making two

\footnotetext{
${ }^{2}$ Correlational analysis (controlling for baseline outcome levels) revealed that compliance with the mindfulness homework exercise was not related to outcomes in the MB group at posttreatment or 1-month follow-up (all $p s \geq .18$ ). We suspect that we did not observe any significant correlations because the brief nature of the mindfulness homework might limit its effectiveness. It is additionally possible that general mindfulness training does not directly affect eating disorder related variables.
} 
comments on 20 different body parts (i.e., hair, skin, eyes, nose, mouth, teeth, chin, neck, shoulders, upper arms, wrists, hands, chest, waist, hips, buttocks, thighs, calves, ankles, and feet), while observing their body in a three-way mirror. A list of these body parts was posted on the outer part of the mirror to guide participants during exposure. Participants were also provided with a suggestion sheet to be used if they had difficulty thinking of comments for a certain body part. These suggestions were specific to each orientation (i.e., positive for the CD condition and neutral for the $\mathrm{NJ}$ and MB conditions).

After explaining the procedure and demonstrating two comments of the appropriate orientation for two different body parts, the experimenter asked the participant to demonstrate the exercise by commenting on two body parts she was comfortable with and one body part she was less comfortable with (i.e., a "hot spot"). Upon successful completion of this practice exercise, the participant was left alone to complete the full exercise. ME was not time-limited. Though all mirror exposure exercises were conducted in private, they were tape recorded and coded by experimenters for adherence. Specifically, experimenters coded adherence to the instructions to comment on each of the 20 listed body parts, to make two different comments about each body part, and to make comments of the appropriate orientation for the condition (i.e., neutral or positive). Adherence to these instructions was rated on a Likert scale ranging from one to four $(1=$ did not complete at all, $2=$ somewhat completed, $3=$ mostly completed, $4=$ fully completed). Due to difficulties with the tape recorder equipment, 6 (3.57\%) of the first $\mathrm{ME}$ sessions and $10(5.95 \%)$ of the second ME sessions did not record properly and were not coded. 
For all of the recorded data, however, adherence was excellent for both the first session $(M=$ 3.97, $S D=0.11)$ and the second session $(M=3.98, S D=0.08){ }^{3}$

Condition-Specific Instructions. Experimenters instructed participants in the intervention procedures by strictly adhering to a script written by the first author in consultation with the third author, a licensed clinical psychologist with expertise in cognitive-behavioral therapies for eating disorders, mindfulness (via training in dialectical behavior therapy) and cognitive dissonance-based prevention. ${ }^{4}$ The mirror exposure scripts for each condition were designed to be as similar as possible, making only the necessary variations for the orientation of each condition. ${ }^{5}$

In the NJ ME participants were instructed to make neutral or descriptive comments (e.g., "this is kind of like describing yourself to someone who is drawing you, but cannot see you"). In the MB ME, participants were instructed to make neutral comments or descriptive comments (same instructions as NJ condition), while additionally trying to maintain a focus of mindfulness (i.e., staying in the present moment, maintaining an open and accepting frame of mind). In the CD ME, participants were instructed to make positive comments during mirror exposure (e.g., "when you are doing this it may help to begin with the phrase, 'I like how my (body area) is

${ }^{3}$ Correlational analysis (controlling for baseline outcome levels) revealed that adherence to the mirror exposure protocol (averaged across sessions one and two) was not related to outcomes at post-treatment or 1-month follow-up (all $p s \geq .15$ ). Since we observed high levels of adherence with little variance, the lack of correlation may be due to a ceiling effect.

${ }^{4}$ The experimental protocol and mirror exposure scripts are available upon request.

${ }^{5}$ In contrast to the CD ME designed by Stice and Presnell (2007), which allowed individuals to make positive comments about their emotional, intellectual, social, and physical qualities, our $\mathrm{CD} \mathrm{ME}$ focused on physically related qualities alone in order to be more congruent with the other conditions. 
[blank]' "). Participants in the CD condition were encouraged to focus as many of their comments as possible on what they liked about the appearance of each body part, though it was anticipated that this might be very difficult due to conflicting social norms. Therefore, participants in the $\mathrm{CD}$ condition were allowed to make positive comments on the function of a body part when they were unable to comment positively about its appearance.

\section{Results}

\section{Preliminary Analyses}

Due to other body image studies taking place at the university (i.e., one with sorority members and one with female athletes), 10 participants were enrolled in other body image programs (e.g., The Female Athlete Body Project, or Reflections: Body Image Program) between the first and second session or first and third session of this study. These participants were evenly distrubuted among conditions (four in $\mathrm{MB}$, three in $\mathrm{NJ}$, and three in $\mathrm{CD}$ ). In an attempt to eliminate this possible confound, analyses (i.e., one-way and mixed models ANOVAs) were run excluding these participants. However, analyses excluding these participants resulted in the same outcomes. Thus, all analyses reported below include these participants.

Missing data was filled in using the multiple inputations function in SPSS. In order to correct for skewness, a square root transformation was used for the BDI-II and the EDE-Q subscale scores (i.e., shape and weight concern, and eating disorder composite) and a logarithmic transform was used on the BCQ, BMI, and age.

A one-way ANOVA indicated that conditions (MB, NJ, CD) did not differ at baseline on any of the dependent measures, BMI, or age (all $p s \geq .17$ ). Due to the change in the recruitment strategy during the third year of the study, we also conducted independent-samples $t$-tests to compare participants recruited during the first and second year of the study with those recruited 
during the third year. We found no significant differences in age, BMI, or outcome measures (at pre-treatment, post-treatment, and 1-month follow-up) in these two groups (all $p s \geq .18$ ), with the exception of age, $t(151)=2.09, p=.04$. Due to the minimal effect size $(d=0.34)$ of this difference in age, the restricted age range of the total sample (17-21 years), and the finding that outcomes were similar for those recruited in years 1 and 2 as compared with those recruited in year 3, we judged that the difference in ages between these two groups is not clinically significant.

To determine the level of body image disturbance in our sample, we compared unadjusted baseline means of body image related outcomes in our sample with means previously found in normal, female college students. Descriptive analyses revealed that the mean scores of our participants on the SBPS (face subscale, $M=4.34, S D=0.79$; body subscale $M=3.74, S D=$ $0.92)^{6}$, EDE-Q shape concern $(M=2.23, S D=1.34)$, EDE-Q weight concern $(M=1.75, S D=$ 1.30), BCQ $(M=47.42, S D=13.35)$, and BIAQ $(M=21.38, S D=7.81$; $)$ were within one standard deviation of the means previously reported on the SBPS (face subscale, $M=4.11, S D=$ 1.06; body subscale, $M=3.16, S D=1.15$; Petrie et al., 2002), EDE-Q shape concern $(M=2.27$, $S D=1.54$; Luce, Crowther, \& Pole, 2008), EDE-Q weight concern $(M=1.97, S D=1.56$; Luce et al., 2008), BCQ $(M=56.0, S D=16$; Reas et al., 2002), and BIAQ $(M=31.5, S D=13.9$; Rosen et al., 1991) in normal, female college students. Thus, our sample is representative of a normal, female, college population.

\footnotetext{
${ }^{6}$ Petrie et al. (2002) used an abbreviated version of the SBPS. They also separated the single item used on the original SBPS to evaluate satisfaction with hips and upper thighs into two separate items. To calculate a means from our sample comparable to theirs, we averaged responses to the items they selected for their body and face subscales. However, consistent with the original version of the SBPS, our scale only had one item addressing hips and upper thighs.
} 


\section{Outcome Analyses}

Main analyses included mixed models ANOVAs with condition (MB, NJ, CD) as a between-subjects factor and time (pre, post, 1-month follow-up) as a within-subjects factor. We used a Bonferroni correction to adjust for multiple comparisons, resulting in a required $p$-level of .008 for significance. To investigate the maintenance of outcomes, we also conducted ANCOVAs, with condition (MB, NJ, CD) as a between-subjects factor and time (post, 1-month follow-up) as a within-subjects factor, using pre-treatment scores as a covariate. As in the main analyses, we again used a Bonferroni correction to adjust for multiple comparisons, resulting in a required $p$-level of .008 for significance.

Mixed models ANOVAS revealed a significant main effect of time and no significant time by condition interactions for the BCQ, BIAQ, BDI-II, shape and weight concern, and eating disorder pathology, such that all conditions led to equal improvement on each of these outcomes (see Table 1 for means, standard deviations, and Cohen's $d$ and Table 2 for results of analyses). In contrast, a main effect for time and a significant time by condition interaction were observed for the SBPS. Post-hoc analyses were conducted to further investigate this interaction. Repeated-measures ANOVAs revealed no significant main effect of time (pre, post, and 1-month follow-up) in the NJ condition, $F(2,108)=.20, p=.822, \eta^{2}=.004$, or the MB condition, $F(2$, $114)=2.86, p=.061, \eta^{2}=.048$. However, a significant main effect of time was observed in the CD condition, such that outcomes improved over time, $F(2,108)=17.65, p<.001, \eta^{2}=.246$.

\section{[INSERT TABLES 1 AND 2 HERE]}

To determine whether the CD condition had significantly better outcomes on the SBPS than both $\mathrm{MB}$ and $\mathrm{NJ}$ conditions, additional post-hoc analyses were run comparing $\mathrm{CD}$ with $\mathrm{MB}$ and $\mathrm{CD}$ with NJ. The mixed models ANOVA with group (CD, MB) and time (pre, post, 1- 
month follow-up) revealed a main effect of time, $F(2,222)=8.93, p<.001, \eta^{2}=.074$, and time by condition interaction, $F(2,222)=11.51, p<.001, \eta^{2}=.094$. The mixed models ANOVA with group (CD, NJ) and time (pre, post, 1-month follow-up) revealed a main effect of time, $F(2$, $216)=6.91, p<.001, \eta^{2}=.060$, and time by condition interaction, $F(2,216)=10.05, p<.001$, $\eta^{2}=.085$. Post-hoc analyses overall indicate that CD produced significantly more improvement in body satisfaction than $\mathrm{MB}$ or $\mathrm{NJ}$.

ANCOVAs, investigating maintenance of treatment gains revealed no main effects for time or significant time by condition interactions (all $p s \geq .04$ ). These results suggest that the benefits of treatment were maintained, though no additional gains were experienced, between post-treatment and 1-month follow-up.

\section{Discussion}

Results partially supported the first hypothesis that MB, NJ and CD MEs would all significantly reduce eating disorder risk factors. Analyses indicated that MB, NJ, and CD ME all improved body checking, body avoidance, depression, shape and weight concern, and eating disorder pathology. The only exception to this finding was the outcome for body satisfaction. Body satisfaction is of unique importance in this study because ME is an intervention specifically designed to target body image disturbance. Analyses of body satisfaction outcomes within each condition indicated that $\mathrm{CD}$ ME was the only condition in which scores on body satisfaction significantly improved over time. This finding is inconsistent with the second hypothesis, that the neutral approach used by NJ and MB MEs would be superior to the positive approach used by CD ME. Results suggest that the positive approach was more effective in improving body satisfaction than the neutral approaches, despite, and possibly because of, its direct opposition to the social norms of fat talk and feminine modesty. 
Results for body satisfaction after MB ME are somewhat consistent with the findings of Delinsky and Wilson (2006). Delinsky and Wilson found that their MB ME improved several eating disorder risk factors that were also improved by the MB ME used in our study, including body checking and avoidance, depression, and shape and weight concern. However, Delinsky and Wilson's MB ME significantly improved body satisfaction as measured by an abbreviated version of the SBPS, whereas the MB ME used in our study did not produce significant improvement on the SBPS. Results for body satisfaction also did not align with previous research on a neutral approach to ME. Two studies investigating a neutral approach, similar to the NJ ME in this study, found that neutral ME improved participants' appearance related selfesteem, a similar outcome to body satisfaction (Hilbert et al., 2002; Jansen et al., 2008).

Conflicting findings may be related to differences in the mirror exposure protocol. For example, clinicians guided the MB ME implemented by Delinsky and Wilson (2006) and the neutral ME implemented by Hilbert et al. (2002), whereas the ME in our study was primarily self-guided, with only brief, scripted instructions from a research assistant. It is possible that having clinicians to guide ME leads to more improvement in body satisfaction when using an MB or NJ approach.

There are several possible explanations as to why CD ME improved body satisfaction significantly more than MB or NJ ME in our study. One reason for differences observed between groups might be due to the possibility that directly opposing the societal norm by verbalizing positive body-related cognitions does in fact create cognitive dissonance, and thereby increases the intervention's ability to change body-related cognitions. Another possibility is that expressing satisfaction as a response on the SBPS reflects a type of positive judgment, which is more consistent with the positive verbalizations used in CD ME than the neutral verbalizations 
used in NJ and MB ME. This explanation might suggest that group differences are due to demand characteristics of the CD condition, rather than changes in body satisfaction. Alternatively, it is possible that this discrepancy reflects that the SBPS measures different aspects of body image than other outcomes such as the shape and weight subscale. Specifically, the shape and weight subscale might measure the level of investment and concern with appearance, while the SBPS measures satisfaction with physical attributes. We also did not find any difference in the effects of each intervention on body checking and avoidance, which are both measures that similarly reflect level of investment in and concern with appearance. Therefore, results may suggest that the positive approach taken by CD ME is a superior intervention for increasing satisfaction, though all of the interventions equally improved overvaluation of appearance.

Interpretation of differences in body satisfaction is somewhat limited by a confounding factor in the CD ME group. Though the CD ME group was encouraged to verbalize positive descriptions related to the appearance of each body part, they were allowed to comment positively on the function of the body part when they could not think of anything positive to say about the appearance of the body part. In contrast, MB and NJ groups commented neutrally on appearance alone. Comments related to function were allowed in the CD condition because it was anticipated that the social norm of feminine modesty and internalization of the thin ideal might make verbalizing two positive thoughts about appearance of each body part too difficult. To elaborate on this study's findings, future studies should compare the effectiveness of verbalization of positive appearance-related cognitions only, to verbalization of positive cognitions related to body appearance and function, during CD ME. It is conceivable that having 
participants focus on functionality helps them gain greater appreciation of what their body can do as opposed to thinking of it simply as an object to be observed.

In addition to the primary outcomes, this study has several other important implications. This is the first study to document the effectiveness of CD ME alone, outside of the context in which CD ME has been previously used, as one of several components of a cognitive dissonance-based eating disorder prevention program called The Body Project (Stice \& Presnell, 2007). Furthermore, this study suggests that ME disseminated by undergraduate-level researchers, and implemented independently, can lead to significant improvement in eating disorder-related variables. The method of dissemination used in this study somewhat parallels the approach taken by Reflections, an empirically supported adaptation of The Body Project designed to be disseminated by trained peer-leaders in sororities (Becker \& Stice, 2008; Becker et al., 2009).

When interpreting the overall findings, it is important to note that we found far more similarities than differences between groups. Though CD ME produced better results on one out of six measures, all versions of ME improved multiple risk factors for eating disorders. Eating disorder prevention programs overall have produced mixed findings for body image improvement and maintenance of the improvement at follow-up (Paxton, 2002; Stice \& Shaw, 2004). Furthermore, interventions that improve body image generally find modest effect sizes (Paxton, 2002). The small effect sizes found in this study are reminiscent of effect sizes previously reported for eating disorder prevention programs (Becker et al., 2006), though the ME intervention used in this study required minimal time and resources in comparison with other programs. Results suggest that supplementing eating disorder prevention and treatment programs with frequent MEs may enhance the effectiveness of these programs. 
Although results are encouraging, conclusions regarding the effectiveness of MEs disseminated by undergraduate-level research assistants are limited due to lack of comparison groups, such as control or clinician-guided ME groups. Results of this study, however, provide solid ground for future research in this area. Additionally, conclusions regarding the effects of each intervention on body image avoidance may be questionable due to the use of the BIAQ. We selected this questionnaire because it is commonly used in research related to body image and was used in previous studies of mirror exposure (Delinsky \& Wilson, 2006; Key et al., 2002). However some of the constructs the BIAQ measures, such as eating restraint and social activities (Rosen et al., 1991), might not be direct indicators of body image avoidance behavior. For example, eating restraint may be a better indicator of overall eating disorder symptoms whereas social activities may be more directly affected by social anxiety.

Our study was further limited in that the $\mathrm{MB}$ and $\mathrm{NJ}$ conditions provided participants with a relatively brief overview of these complex concepts. For example, our MB condition primarily focused on maintaining awareness of the present moment and refraining from judgment, whereas mindfulness skills as taught in other therapeutic protocols emphasize additional aspects of mindfulness, such as observation and acceptance (Lynch, Chapman, Rosenthal, Kuo, \& Linehan, 2006). The MB condition was also weaker than a typical MB intervention because $\mathrm{MB}$ interventions would typically devote more time to cultivating an understanding of mindfulness before attempting to apply it to an anxiety-provoking exercise such as ME. Future studies might investigate the effectiveness of incorporating more thorough mindfulness training leading up to $\mathrm{MB} \mathrm{ME}$, especially mindfulness training as related to the body and self. Similarly to the MB condition, the presentation of NJ was limited in our study, focusing only on the idea of remaining objective. Participants did not receive training that 
adequately addressed the complexities of being nonjudgmental as it is understood from the perspective of mindfulness. From this viewpoint, being completely nonjudgmental would not just include being objective; it would also require other aspects of mindfulness (e.g., an attitude of openness and acceptance toward what is objectively observed). Thus, interpretation of the effectiveness of MB and NJ approaches based on our findings should be limited to these concepts, as they have been operationalized in this study. Results overall might be more reflective of the use of a neutral stance during $\mathrm{ME}$ (MB and NJ conditions) as compared with the use of a positive stance during $\mathrm{ME}$ (CD condition).

Conclusions about effectiveness should also be tempered by the fact that most effect sizes observed in this study were generally small. Effect sizes might be enhanced if these mirror exposures were tested in an eating disordered population, or even in a non-clinical population with higher levels of body dissatisfaction. Though we sought to recruit individuals with body image disturbance by advertising the study as a program for improving body image, comparison of our sample with norms revealed that our sample exhibited normal outcome levels at baseline. Because we were unsuccessful in recruiting individuals with above average levels of body image disturbance, we may have encountered a ceiling effect that led to small effect sizes. Effect sizes might additionally improve by increasing the frequency or duration of mirror exposure. Future studies might also investigate whether effectiveness of ME is enhanced when ME is guided by a clinician (Delinsky \& Wilson, 2006; Hilbert et al., 2002).

\section{Conclusions}

Results of this study overall suggest that MB, NJ, and CD MEs each improve several eating disorder-related outcomes in a non-clinical, female sample. However, results also suggest that only CD ME significantly improves body satisfaction. Though significant improvement was 
observed in each condition, effect sizes generally were small. Future research should investigate whether greater effect sizes are observed with an increased frequency or duration of mirror exposure, or with a sample exhibiting above-average levels of body dissatisfaction. Follow-up studies might additionally compare the effectiveness of MEs disseminated by undergraduates with MEs guided by a clinician, and explore whether our findings can be replicated in a clinical sample. 


\section{Acknowledgements}

We would like to acknowledge and thank Kristin Prasifka and Anjoli Reed for their valuable assistance with completing this study. This study was funded by the Mach Family

Fellowship at Trinity University and by Trinity University's Howard Hughes Medical Institute Undergraduate Research Program.

\section{Role of the Funding Source}

The funding sources had no involvement in the course of the study or its publication. 


\section{References}

American Psychiatric Association (2000). Diagnostic and statistical manual of mental disorders: $D S M-I V-T R\left(4^{\text {th }}\right.$ ed. $)$. Washington, D.C.: Author.

Beck, A. T., Steer, R. A., \& Brown, G. K. (1996). Manual for the Beck Depression Inventory-II. San Antonio, TX: Psychological Corporation.

Becker, C. B., Bull, S., Schaumberg, K., Cauble, A., \& Franco, A. (2008). Effectiveness of peerled eating disorders prevention: A replication trial. Journal of Consulting and Clinical Psychology, 76, 347-354.

Becker, C. B., Smith, L. M., \& Ciao, A. C. (2006). Peer-facilitated eating disorder prevention: A randomized effectiveness trial of cognitive dissonance and media advocacy. Journal of Counseling Psychology, 53, 550-555.

Becker, C. B., \& Stice, E. (2008). Sorority body image program: Group leader guide. New York, NY: Oxford University Press.

Becker, C. B., Stice, E., Shaw, H., \& Woda, S. (2009). Use of empirically supported interventions for psychopathology: Can the participatory approach move us beyond the research-to-practice gap? Behaviour Research and Therapy, 47, 265-274.

Becker, C. B., Wilson, C., Williams, A., Kelly, M., McDaniel, L., \& Elmquist, J. (2010). Peerfacilitated cognitive dissonance versus healthy weight eating disorders prevention: A randomized comparison. Body Image, 7, 280-288.

Berscheid, E., Walster, E., \& Bohrnstedt, G. (1973). Body image: the happy American body: A survey report. Psychology Today, 7, 119-131.

Britton, L. E., Martz, D. M., Bazzini, D. G., Curtin, L. A., \& LeaShomb, A. (2006). Fat talk and self presentation of body image: Is there a social norm for women to self-degrade? Body 
Image, 3, 247-254.

Calugi, S., Dalle Grave, R., Ghisi, M., \& Sanavio, E. (2006). Validation of the body checking questionnaire (BCQ) in an eating disorders population. Behavioural and Cognitive Psychotherapy, 34, 233-242.

Delinsky, S. S., \& Wilson, G. T. (2006). Mirror exposure for the treatment of body image disturbance. International Journal of Eating Disorders, 39, 108-116.

Dozois, D. J. A., Dobson, K. S., \& Ahnberg, J. L. (1998). A psychometric evaluation of the Beck Depression Inventory-II. Psychological Assessment, 10, 83-89.

Fairburn, C. G., \& Beglin, S. J. (1994). Assessment of eating disorders: Interview or self-report questionnaire? International Journal of Eating Disorders, 16, 363-370.

Fairburn, C. G., Peveler, R. C., Jones, R., Hope, R. A., \& Doll, H. A. (1993). Predictors of 12month outcome in bulimia nervosa and influence of attitudes to shape and weight. Journal of Consulting and Clinical Psychology, 61, 696-698.

Fairburn, C. G., Stice E., Cooper, Z., Doll, H. A., Norman, P. A., \& O’Connor, M. E. (2003). Understanding persistence in bulimia nervosa: A 5-year naturalistic study. Journal of Consulting and Clinical Psychology, 71, 103-109.

Freeman, R., Beach, B., Davis, R., \& Solym, L. (1985). The prediction of relapse in bulimia nervosa. Journal of Psychiatric Research, 19, 349-353.

Hilbert, A., \& Tuschen-Caffier B. (2005). Body-related cognitions in binge-eating disorder and bulimia nervosa. Journal of Social and Clinical Psychology, 24, 561-579.

Hilbert, A., Tuschen-Caffier, B., \& Vögele (2002). Effects of prolonged and repeated body image exposure in binge-eating disorder. Journal of Psychosomatic Research, 52, 137- 
144.

Jansen, A., Bollen, D., Tuschen-Caffier, B., Roefs, A., Tanghe, A., \& Braet, C. (2008). Mirror exposure reduces body dissatisfaction and anxiety in obese adolescents: A pilot study. Appetite, 51, 214-217.

Key, A., George, C. L., Beattie, D., Stammers, K., Lacey, H., \& Waller, G. (2002). Body image treatment within an inpatient program for anorexia nervosa: The role of mirror exposure in the desensitization process. International Journal of Eating Disorders, 13, 185-190.

Killen, J. D., Taylor, C. B., Hayward, C., Haydel, K. F., Wilson, D. M., Hammer, L., ... Strachowski, D. (1996). Weight concerns influence the development of eating disorders: A 4-year prospective study. Journal of Consulting and Clinical Psychology, 64, 936-940.

Luce, K. H., Crowther, J. H., \& Pole, M. (2008). Eating Disorder Examination Questionnaire (EDE-Q): Normal for undergraduate women. International Journal of Eating Disorders, 41, 273-276.

Lynch, T. R., Chapman, A. L., Rosenthal, M. Z., Kuo, J. R., \& Linehan. M. M. (2006). Mechanisms of change in dialectial behavioral therapy: Theoretical and empirical observations. Journal of Clinical Psychology, 62, 459-480.

National Institutes of Health. (1998). Clinical guidelines on the identification, evaluation, and treatment of overweight and obesity in adults: The evidence report (NIH Publication No. 98-4083). Washington, DC: U.S. Department of Health and Human Services.

Nichter, M. (2000). Fat talk: What girls and their parents say about dieting. Cambridge, MA: Harvard University Press.

Peterson, C. B., Crosby, R. D., Wonderlich, S. A., Joiner, T., Crow, S. J., Mitchell, J. E., ... le Grange, D. (2007). Psychometric properties of the eating disorder examination- 
questionnaire: Factor structure and internal consistency. International Journal of Eating Disorders, 40, 386-389.

Paxton., S. J. (2002, July). Research review of body image programs: An overview of body image prevention interventions. Melbourne, VIC: Victorian Government Department of Human Services. Retrieved from the Victorian Government Health Information Web site: http://www.health.vic.gov.au/healthpromotion/evidence_res/body_image.htm

Petrie, T. A., Tripp, M. M., \& Harvey, P. (2002). Factorial and construct validity of the body parts satisfaction scale-revised: An examination of minority and nonminority women. Psychology of Women Quarterly, 26, 213-221.

Reas, D. L., Whisenhunt, B. L., Netemeyer, R., \& Williamson, D. A. (2002). Development of the body checking questionnaire: A self-report measure of body checking behaviors. International Journal of Eating Disorders, 31, 324-333.

Rosen, J. (1990). Body-image disturbance in eating disorders. In T. F. Cash \& T. Pruzinsky (Eds.), Body images: Development, deviance, and change (pp. 190-216). New York, NY: The Guilford Press.

Rosen, J. C., Srebnik, D., Saltzberg, E., \& Wendt, S. (1991). Development of a body image avoidance questionnaire. Psychological Assessment: A Journal of Consulting and Clinical Psychology, 3, 32-37.

Rudman, L. A. (1998). Self-promotion as a risk factor for women: The costs and benefits of counterstereotypical impression management. Journal of Personality and Social Psychology, 74, 629-645.

Salzberg, S., \& Goldstein, J. (2001). Breathing meditation. On Insight meditation [CD]. Boulder, CO: Sounds True. 
Stewart, T. M. (2004). Light on body image treatment: Acceptance through mindfulness. Behavior Modification, 28, 783-811.

Stice, E. (2002). Risk and maintenance factors for eating pathology: A meta-analytic review. Psychological Bulletin, 128, 825-848.

Stice, E., \& Presnell, K. (2007). The body project: Promoting body acceptance and preventing eating disorders: Facilitator guide. New York, NY: Oxford University Press.

Stice, E., \& Shaw, H. (2004). Eating disorder prevention programs: A meta-analytic review. Psychological Bulletin, 130, 206-227.

Stice, E., Shaw, H., Becker, C. B., \& Rohde, P. (2008). Dissonance-based interventions for the prevention of eating disorders: Using persuasion principles to promote health. Prevention Science, 9, 114-128.

Vocks, S., Legenbauer, T., Wächter, A., Wucherer, M., \& Kosfelder, J. (2007). What happens in the course of body exposure? Emotional, cognitive, and physiological reactions to mirror confrontation in eating disorders. Journal of Psychosomatic Research, 62, 231-239.

Widenauer, U., Lennerts, W., Talbot, P., Touyz, S. W., \& Beumont, P. J. (1993). How well are 'cured' anorexia nervosa patients? An investigation of 16 weight-recovered anorexic patients. British Journal of Psychiatry, 163, 195-200.

Wiebe, J. S., \& Penley, J. A. (2005). A psychometric comparison of the Beck Depression Inventory-II in English and Spanish. Psychological Assessment, 17, 481-485.

Wilson, G. T., \& Fairburn, C. G. (2002). Treatments for eating disorders. In P. E. Nathan \& J. M Gorman (Eds.), A guide to treatments that work ( $2^{\text {nd }}$ ed., pp. 559-592). New York, NY: Oxford University Press. 
Table 1. Means and Standard Deviations for Dependent Measures.

\begin{tabular}{cccccc}
\hline & & $\begin{array}{c}\text { Post } \\
\text { Treatment }\end{array}$ & $\begin{array}{c}\text { 1-month } \\
\text { Follow-up }\end{array}$ & $\begin{array}{c}\text { Post } \\
\text { Treatment }\end{array}$ & $\begin{array}{c}\text { 1-month } \\
\text { Follow-up }\end{array}$ \\
\cline { 2 - 5 } Measures & $M(S D)$ & $M(S D)$ & $M(S D)$ & $d$ & $d$
\end{tabular}

Body Checking Questionnaire

$\begin{array}{llllll}\text { MB } & 47.75(15.96) & 47.37(16.65) & 45.27(16.78) & 0.02 & 0.15 \\ \text { NJ } & 46.84(12.36) & 44.98(13.42) & 43.99(13.40) & 0.14 & 0.22 \\ \text { CD } & 47.66(11.34) & 44.66(11.15) & 42.22(10.59) & 0.27 & 0.50\end{array}$

Body Image Avoidance Questionnaire

$\begin{array}{llllll}\text { MB } & 22.03(9.34) & 21.37(9.71) & 20.21(10.38) & 0.07 & 0.18 \\ \text { NJ } & 19.85(7.02) & 18.57(7.00) & 18.75(7.47) & 0.18 & 0.15 \\ \text { CD } & 22.22(6.62) & 20.90(6.56) & 20.13(6.83) & 0.20 & 0.31\end{array}$

Satisfaction with Body Parts Scale

$\begin{array}{llllll}\text { MB } & 98.92(15.45) & 97.76(17.36) & 100.21(16.66) & 0.07 & 0.08 \\ \text { NJ } & 101.43(11.55) & 100.78(12.36) & 101.25(13.52) & 0.05 & 0.01 \\ \text { CD } & 99.00(12.37) & 104.76(12.48) & 103.48(12.02) & 0.46 & 0.37\end{array}$

Beck Depression Inventory-II

$\begin{array}{llllll}\text { MB } & 9.88(7.70) & 9.87(9.07) & 8.76(8.85) & 0.001 & 0.14 \\ \text { NJ } & 8.24(5.45) & 8.07(5.64) & 6.98(5.32) & 0.03 & 0.23 \\ \text { CD } & 8.38(5.72) & 7.28(6.07) & 6.91(5.97) & 0.19 & 0.25\end{array}$

EDE-Q Shape and Weight Concern Subscale

$\begin{array}{llllll}\text { MB } & 1.99(1.40) & 1.79(1.36) & 1.61(1.39) & 0.14 & 0.27 \\ \text { NJ } & 1.94(1.21) & 1.68(1.13) & 1.51(1.08) & 0.22 & 0.37 \\ \text { CD } & 2.03(1.21) & 1.95(1.13) & 1.71(1.11) & 0.07 & 0.28\end{array}$

EDE-Q Eating Disorder Pathology Composite
MB
11.34(7.99)
9.95(7.98)
8.48(8.04)
0.17
0.36 


$\begin{array}{llllll}\text { NJ } & 11.40(7.30) & 9.40(6.57) & 8.69(7.45) & 0.29 & 0.37 \\ \text { CD } & 10.64(6.84) & 9.98(6.28) & 8.22(5.64) & 0.10 & 0.39\end{array}$

Note: Mindfulness-based (MB) $n=58$, Nonjudgmental (NJ) $n=55$, Cognitive Dissonance (CD) $n=55$. 
Table 2. Mixed models ANOVA Main Outcome Analyses

$\begin{array}{lll}\text { Measures Time } & \text { Time x Condition }\end{array}$

Body Checking Questionnaire

$$
F(2,330)=37.30, p<.001, \eta^{2}=.184 F(4,330)=2.52, p=.041 \eta^{2}=.030
$$

Body Image Avoidance Questionnaire

$$
F(2,330)=13.55, p<.001, \eta^{2}=.076 \quad F(4,330)=.91, p=.458, \eta^{2}=.011
$$

Satisfaction with Body Parts Scale

$$
F(2,330)=5.12, p=.006, \eta^{2}=.030 \quad F(4,330)=7.39, p<.001, \eta^{2}=.082
$$

Beck Depression Inventory-II

$$
F(2,330)=12.74, p<.001, \eta^{2}=.072 \quad F(4,330)=.48, p=.748, \eta^{2}=.006
$$

EDE-Q Shape and Weight Concern Subscale

$$
F(2,330)=35.15, p<.001, \eta^{2}=.176 \quad F(4,330)=.83, p=.510, \eta^{2}=.010
$$

EDE-Q Eating Disorder Pathology Composite

$$
F(2,330)=41.15, p<.001, \eta^{2}=.200 F(4,330)=.99, p=.413, \eta^{2}=.012
$$

Note: Mindfulness-based (MB) $n=58$, Nonjudgmental (NJ) $n=55$, and Cognitive Dissonance (CD) $n=55$. 